Article

\title{
A Thermal and Nanomechanical Study of Molecular Crystals as Versatile Mocks for Pentaerythritol Tetranitrate
}

\author{
Alexandra C. Burch ${ }^{1,2}$, Zakary R. Wilde ${ }^{3}\left(\mathbb{D}\right.$, David F. Bahr $^{1}{ }^{1}$ and John D. Yeager ${ }^{2, *(1)}$ \\ 1 School of Materials Engineering, Purdue University, West Lafayette, IN 47907, USA; \\ aburch@lanl.gov (A.C.B.); dfbahr@purdue.edu (D.F.B.) \\ 2 High Explosives Science and Technology, Los Alamos National Laboratory, Los Alamos, NM 87545, USA \\ 3 Detonation Science and Technology, Los Alamos National Laboratory, Los Alamos, NM 87545, USA; \\ zwilde@lanl.govV \\ * Correspondence: jyeager@lanl.gov; Tel.: +1-505-665-0879
}

Received: 31 January 2020; Accepted: 14 February 2020; Published: 19 February 2020

\begin{abstract}
Pentaerythritol tetranitrate (PETN) is a commonly used high explosive (HE) in detonators. Often, surrogate or "mock" materials are used in place of HE for mechanical tests, proofing out equipment, or developing new diagnostics. However, there is no commonly accepted mock for PETN. A good mock should match at least one physical property of the target material, and ideally mimic multiple thermal and mechanical properties. Here, we investigate several molecular crystals to evaluate their efficacy in mocking PETN density, melting point, elastic modulus, hardness, plastic deformation, and fracture behavior. Materials were tested with a combination of calorimetry and nanoindentation. Two materials, 2,4,6-trifluorobenzoic acid (246 TFBA) and mesoerythritol, were downselected for detailed indentation study after the initial round of screening experiments, both were found to mimic PETN mechanical behavior quite well, 246 TFBA closer to PETN in most properties (hardness, modulus, and density) than erythritol, but erythritol having advantages in relative cost and matching the onset of yield. Depending on the desired implementation of the mock, one material may be preferred over the other, but both have potential as generic mocks for PETN. Nanoindentation is demonstrated as a versatile tool to provide rapid screening of these materials' mechanical properties.
\end{abstract}

Keywords: nanoindentation; explosive; mock; fracture

\section{Introduction}

Pentaerythritol tetranitrate (PETN) is a high explosive (HE) that is generally accepted to be the most sensitive of secondary explosives, with any explosive more sensitive than PETN being categorized as a primary explosive [1]. PETN is most commonly used as a detonator material due to its reliable initiation, and finds some limited applications in composite explosives as well [2]. While PETN can be safely handled in most situations, its relatively high sensitivity to impact and friction can pose some hazards. Occasionally, it can be appropriate to replace PETN with a non-hazardous surrogate material ("mock") for initial experiments. These could include proofing equipment used for making detonators, performing thermal stability/aging experiments, performing mechanical experiments, or developing new diagnostic capabilities. The goal is to carry out the desired test on the mock instead of the HE, having a reasonable expectation that the results of the experiment would be similar for both materials.

There has been limited investigation in the literature for suitable mocks for PETN, in recent years using mocks to develop techniques for explosives trace detection [3-5]. For other HE materials, inert molecular crystals are often used as mocks in cases where handling HE is a concern [6]. A high fidelity 
or versatile mock will replicate the most important property of the target HE (e.g., density) while also mimicking as many of the other thermal and mechanical properties of the HE as possible. While there are many methods to identify suitable mocks, generally they fall along two ideas: (1) use an inert material with similar molecular structure to the HE [7-9]; (2) search for inert materials that mimic a specific property, and then choose target materials based on molecular structure or other secondary properties [10-12]. For PETN specifically, our properties of interest include density, melting point, elastic properties, and fracture behavior.

When searching the literature for candidate mock materials, density and thermal properties are often reported along with other routine characterizations of a material. Mechanical properties, on the other hand, are usually not reported for molecular crystals because they are either proprietary materials (e.g., pharmaceuticals) or not of substantial interest in the application. Nanoindentation can be a powerful tool to measure mechanical properties when testing potential mocks because measurements can be performed quickly with high repeatability on extremely small quantities of material. This also lends itself well to the testing of explosives, where the risk associated with handling the material is proportional to the amount of material present. Nanoindentation has previously been used for the purpose of characterizing explosives such as cyclotetramethylene-tetranitramine (HMX), cyclotrimethylenetrinitramine (RDX), triaminotrinitrobenzene (TATB), and others [13-17]. PETN had previously been characterized via nanoindentation but some unusual properties were discovered, such as extreme depth dependence of the elastic modulus [18], which merited a re-examination for this present work.

Here, we report the results of several thermal and mechanical tests on PETN and newly identified mock candidates. We developed selection criteria for PETN and identified five candidates through a combination of Cambridge Structural Database (CSD) [19] queries and general literature searching. Meso-erythritol (erythritol), 2,3,4,5,6-pentafluorobenzamide (PFBA), 2,4,6-trifluorobenzoic acid (246 TFBA), 1,1,1,5,5,5-hexafluoropentane-2,2,4,4-tetraol (HFPT), and $\mathrm{N}, \mathrm{N}^{\prime}$-bis(2,3,4,5,6-pentafluorophenyl)oxamide (N-BPFPO) were chosen for examination as potential thermomechanical mocks for PETN. Mechanical comparisons were initially made on the basis of hardness and elastic modulus, while thermal comparisons used differential scanning calorimetry (DSC) to determine properties such as melting point. These initial tests enabled downselection to two candidates, which were then subjected to intensive nanoindentation studies for determining incipient plastic deformation, indentation fracture toughness, and in-plane anisotropy in elastic modulus. We find erythritol and 246 TFBA to be particularly promising for mocking PETN in various scenarios, with respective advantages and disadvantages that may result in selection for specific applications. Quantitative and qualitative comparisons of the mocks to PETN are given and the merits of each are discussed.

\section{Materials and Methods}

All materials except for N-BPFPO and PETN were purchased commercially and used without further purification. None of the materials were initially suitable for nanoindentation as received, generally presenting as fine powders, and so single crystals were crystallized from appropriate solvents. Meso-erythritol (Erythritol) was purchased from Sigma-Aldrich (now Millipore Sigma) and crystals were grown from a water solution. Pentafluorobenzamide (PFBA) was purchased from Alfa Aesar and crystallized from dichloromethane solution. 1,1,1,5,5,5-hexafluoropentane-2,2,4,4-tetraol (HFPT) was purchased from SynQuest Laboratories (Alachua, FL) and crystallized from acetone. 2,4,6-trifluorobenzoic acid (246 TFBA) was purchased from TCI America and crystallized from acetone. N-BPFPO was synthesized using literature methods [20], reacting pentafluoroaniline (Sigma-Aldrich) with oxalyl chloride (Acros Organics) in benzene. The mixture was concentrated under vacuum and recrystallized in a 1:1 solution of ethyl acetate:hexanes. Fine crystalline powder was recovered, and larger crystals suitable for nanoindentation were produced by slow recrystallization from an acetone solution. PETN was synthesized at and provided by Purdue University and single crystals were 
crystallized in isopropyl alcohol. Figure 1 shows optical micrographs of the indentation surface of the six materials, and Figure 2 shows the chemical structure of each of the six materials.
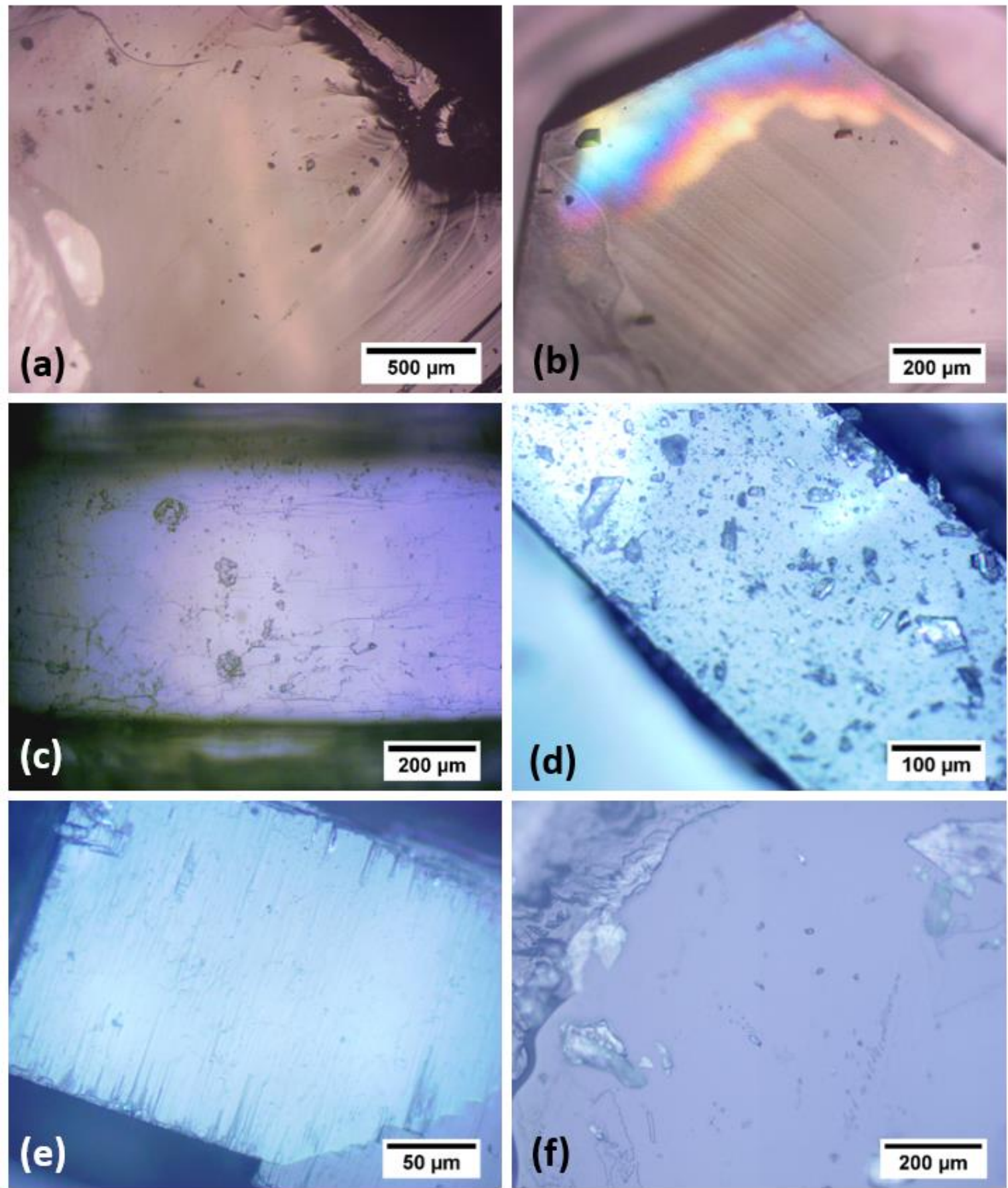

Figure 1. Optical micrographs of single crystals of (a) meso-erythritol (erythritol), (b) pentaerythritol tetranitrate (PETN), (c) 1,1,1,5,5,5-hexafluoropentane-2,2,4,4-tetraol (HFPT), (d) $\mathrm{N}, \mathrm{N}^{\prime}$-bis(2,3,4,5,6-pentafluorophenyl)oxamide (N-BPFPO), (e) pentafluorobenzamide (PFBA), and (f) 2,4,6-trifluorobenzoic acid (246 TFBA). 
<smiles>OC[C@@H](O)[C@H](O)CO</smiles>

(a)<smiles>OC(O)(CF)CC(O)(O)CF</smiles>

(c)

(e)<smiles>NC(=O)c1c(F)c(F)c(F)c(F)c1F</smiles>

(b)<smiles>O=[N+]([O-])OCC(CO[N+](=O)[O-])(CO[N+](=O)[O-])CO[N+](=O)[O-]</smiles><smiles>O=C(Nc1c(F)c(F)c(F)c(F)c1F)C(=O)Nc1c(F)c(F)c(F)c(F)c1F</smiles>

(d)<smiles>O=C(O)c1c(F)cc(F)cc1F</smiles>

(f)

Figure 2. Chemical structures of (a) erythritol, (b) PETN, (c) HFPT, (d) N-BPFPO, (e) PFBA, and (f) 246 TFBA.

All samples were mounted using the technique described by Maughan et al. [21] for mounting small crystals in which single crystals on a level surface are glued to a 7-mm steel disc from above. Nanoindentation was performed using a Hysitron Triboindenter 950, with both Berkovich and cube corner indenter probes on both low-load and high-load transducers, with the low-load transducer used for all indents below a peak load of $12 \mathrm{mN}$. All indents were quasistatic open-loop with $30 \mathrm{~s}$ loading, $5 \mathrm{~s}$ hold, and $5 \mathrm{~s}$ unload times, a loading profile that has previously been used to characterize other molecular crystals $[13,15]$. All indents for the purpose of determining hardness and elastic modulus had a peak load of $1 \mathrm{mN}$ and the unloading curves were analyzed using the Oliver and Pharr technique [22]. Indents performed for the purpose of quantifying indentation fracture threshold were analyzed using the technique developed by Morris et al. [23] with material-specific procedures described in a previous publication by the authors [24].

Of the $1 \mathrm{mN}$ indents used to determine hardness and elastic modulus, 112 indents were performed on six PETN crystals, 33 indents on four 246 TFBA crystals, 26 indents on four PFBA crystals, 8 indents on two HFPT crystals, 19 indents on three N-BPFPO crystals, and 181 indents on 13 erythritol crystals. Of the indents at various loads used to determine indentation fracture behavior, 80 indents were performed on five PETN crystals, 20 indents on three 246 TFBA crystals, and 52 indents on four erythritol crystals.

DSC was performed on PETN and the five mock candidates in triplicate using a heating rate of $10{ }^{\circ} \mathrm{C} / \mathrm{min}$. Each sample was run from 40 to $400{ }^{\circ} \mathrm{C}$ using a Q2000 DSC (TA Instruments, New Castle, DE) that had been calibrated with an indium standard.

\section{Results}

\subsection{Material Selection}

The general methodology for creating a list of mock candidates and then downselecting to several materials for further testing has been reported for HMX recently [25]. Following that example, five materials were chosen as potential mock candidates for PETN: 246 TFBA, PFBA, HFPT, N-BPFPO, and 
erythritol. Baseline properties for each of these materials including PETN are listed in Table 1. The initial selection criteria were that the materials be inert molecular crystals with density and crystal structure similar to PETN. The first four mocks had similar or slightly higher density, but had a more anisotropic crystal structure. Erythritol, while much lower in density, has the same crystal structure as PETN [26] and is a precursor material in the synthesis process for the related energetic material erythritol tetranitrate [27].

Table 1. Physical property values used as initial mock selection criteria for PETN and each of the materials initially under consideration as mechanical mocks for PETN. References contain complete X-ray data.

\begin{tabular}{ccc}
\hline Material & Density $\mathbf{( g / \mathbf { c m } ^ { 3 } )}$ & Crystal Structure \\
\hline PETN & 1.778 & Tetragonal [28] \\
\hline 246 TFBA & 1.759 & Monoclinic [29] \\
\hline Erythritol & 1.452 & Tetragonal [30] \\
\hline HFPT & 1.941 & Monoclinic [31] \\
\hline N-BPFPO & 1.932 & Monoclinic [20] \\
\hline PFBA & 1.884 & Monoclinic [32]
\end{tabular}

\subsection{Thermal Characterization}

Figure 3 shows typical DSC results for each of the six materials tested, with the thermal profiles offset along the y-axis for clarity. PETN has been extremely well-characterized thermally in the literature and so is only shown here for reference when assessing desired mock performance and confirmation that the measurements were done correctly.

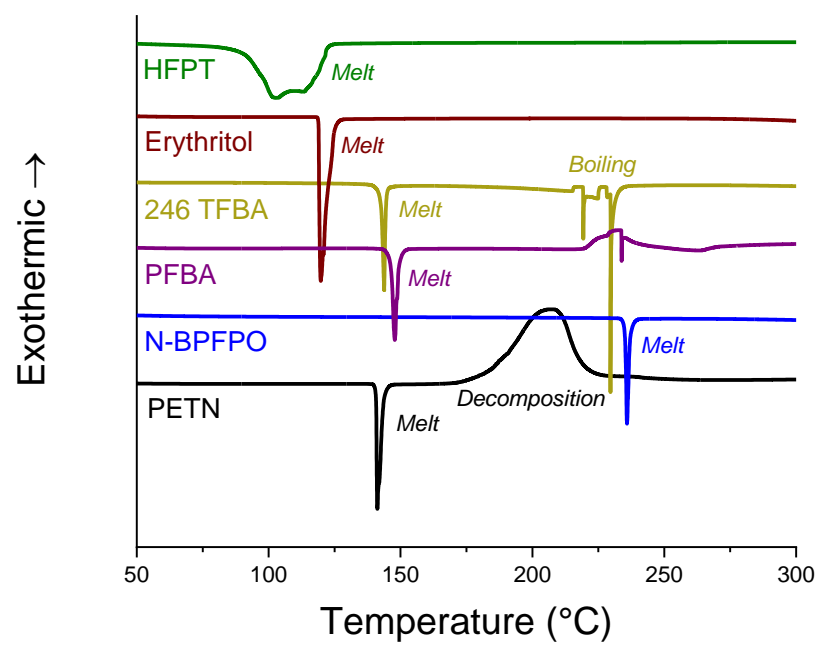

Figure 3. Typical differential scanning calorimetry (DSC) thermal profiles for PETN and mock candidates. Profiles are arbitrarily offset along the y-axis for clarity.

These results show that several of the mock materials exhibit thermal behavior within acceptable margins to mimic PETN. The average melting points of each material (from three measurements), defined for convenience here as the onset of the endotherm, are given in Table 2. HFPT showed evidence of sublimation as low as $50^{\circ} \mathrm{C}$, with onset of melt around $93^{\circ} \mathrm{C}$, and so is not a good thermal match for PETN. N-BPFPO melts at a much higher temperature than PETN, which could be useful in some applications, but is not ideal for a thermally similar mock. The other materials are reasonably similar, with 246 TFBA being nearly identical to PETN. 
Table 2. Thermal properties of interest for PETN and mock candidates.

\begin{tabular}{cc}
\hline Material & Melt Temperature $\left({ }^{\circ} \mathbf{C}\right)$ \\
\hline PETN & 141 \\
\hline 246 TFBA & 143 \\
\hline Erythritol & 119 \\
\hline HFPT & 93 \\
\hline N-BPFPO & 235 \\
\hline PFBA & 147 \\
\hline
\end{tabular}

\subsection{Hardness and Modulus Measurements}

Each of the six materials previously discussed was indented with a Berkovich indenter probe with an open-loop load function to a nominal load of approximately $1000 \mu \mathrm{N}$; a representative load-depth curve for each material is shown in Figure 4.

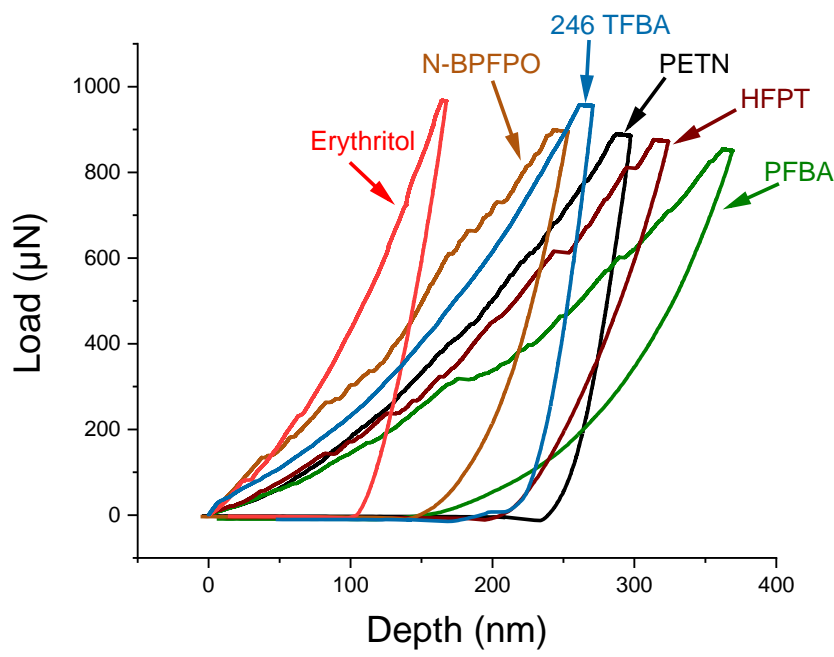

Figure 4. Typical load-depth curves for PETN and each of the materials initially under consideration as mechanical mocks for PETN.

Load-depth data from several such indents per material were analyzed using the method described by Oliver and Pharr [22] to obtain elastic modulus and hardness values, averages for which are reported for each material in Table 3.

Table 3. Average values for elastic modulus and hardness, as measured via nanoindentation, for PETN and each of the materials initially under consideration as mechanical mocks for PETN.

\begin{tabular}{ccc}
\hline Material & Elastic Modulus (GPa) & Hardness (GPa) \\
\hline PETN & $15.2 \pm 2.4$ & $0.45 \pm 0.08$ \\
\hline 246 TFBA & $19.2 \pm 2.6$ & $0.54 \pm 0.18$ \\
\hline Erythritol & $21.2 \pm 2.4$ & $1.22 \pm 0.13$ \\
\hline HFPT & $6.3 \pm 0.4$ & $0.33 \pm 0.07$ \\
\hline N-BPFPO & $17.6 \pm 0.4$ & $0.44 \pm 0.07$ \\
\hline PFBA & $5.8 \pm 1.5$ & $0.24 \pm 0.03$
\end{tabular}

Nanoindentation results for PETN were previously published by Zhai and McKenna [18], showing a dramatic depth dependence for elastic modulus. Their results showed that PETN appeared to be 
stiffer (i.e., higher modulus) at the surface, with a significantly decreasing stiffness as a function of depth (indenter penetration) into the material. This type of result is atypical for similar molecular crystals, both energetic and inert, that have previously been characterized via nanoindentation. To assess the elastic modulus of PETN crystals used in this study, indentations were made and modulus measured over a similar range of depths, as shown in Figure 5. No such depth dependence was seen, with the experiment repeated over a period of seven months to ensure thoroughness. While no depth dependence was revealed, the elastic modulus of these PETN crystals did appear to decrease from month 0 to month 7, prompting additional assessment of the elastic modulus again at month 13 to observe if any changes were occurring due to the age of the material. While the elastic modulus of the newly synthesized material measured $18.2 \pm 2.8 \mathrm{GPa}$, the material at ages 7 months and 13 months appears to have settled around 15.0-15.8 GPa. Due to the behavior of the samples at larger depths at month 0 and the low indentation depths of typical nanoindentation tests, differences over time were also considered only for indents below $300 \mathrm{~nm}$ in depth. Considering only those indents below a depth of $300 \mathrm{~nm}$ had a minimal effect on the results, with the elastic modulus at month 0 measuring $18.4 \pm 2.9 \mathrm{GPa}$, at month 7 measuring $15.1 \pm 0.6 \mathrm{GPa}$, and at month 13 measuring $15.8 \pm 1.8 \mathrm{GPa}$, unchanged due to the lack of higher-depth indents at month 13. Other studies on PETN powder have shown similar trends with properties such as surface area coarsening, where newly synthesized PETN initially coarsens at a very high rate but the rates soon become much more gradual, if not completely stable $[33,34]$.

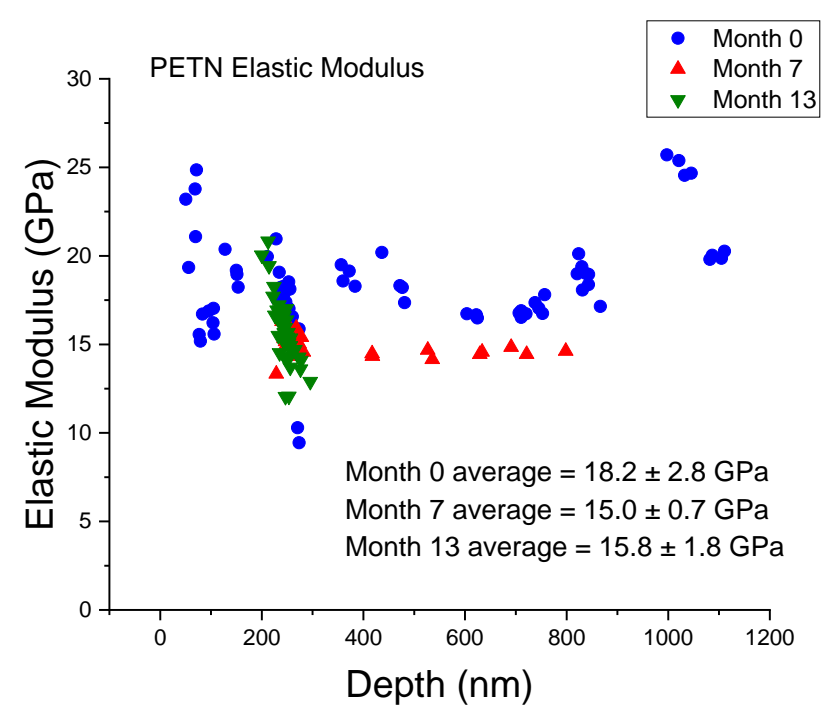

Figure 5. Elastic modulus of PETN as a function of maximum indentation depth. This material does not show any significant depth dependence of elastic modulus. Elastic modulus of the newly synthesized material (month 0 ) is higher than later measurements (month 7 , month 13) but as the material ages the modulus appears to stabilize. This is consistent with observation of other properties of PETN as a function of time (reference).

Following these initial test results, evaluation of PFBA, HFPT, and N-BPFPO as mock candidates for PETN was halted. N-BPFPO did match the modulus and hardness of PETN quite well, but since the density and melting points were much higher it was not considered as good a candidate as 246 TFBA and erythritol. Another practical consideration was that there is no current commercial supplier of N-BPFPO, requiring local synthesis to produce material. However, the synthesis is not overly complicated, so future examination of this molecule as a mock for high density or high melting point explosives is probably warranted. While closer in density and melting temperature, PFBA and HFPT both had significantly lower elastic modulus and hardness than PETN. Detailed mechanical testing was only continued on PETN, erythritol, and 246 TFBA. 


\subsection{Incipient Plasticity and Fracture Behavior}

Incipient plasticity and fracture behavior of erythritol and 246 TFBA were studied in comparison to PETN because PETN powder is subjected to high mechanical loads when pressed into pellets for detonators. During compaction of PETN, the mechanical properties being probed include elastic, plastic, and fracture responses. Nanoindentation can provide detailed information on each of these regimes of mechanical response. Elastic behavior was reported in the previous section, as was hardness (resistance to plastic deformation). Material hardness is effectively dominated by the resistance to the motion of dislocations (i.e., plastic flow). Study of the initiation of dislocations can be particularly impactful when deforming either reasonably defect-free samples or materials with limited slip systems [16,35], both of which describe the molecular single crystals tested here. The onset, or incipient, plasticity can be assessed by "pop-in" analysis. A pop-in is a sudden increase in displacement without a corresponding increase in applied load commonly observed in indentation systems that are operated primarily in load control, and the first pop-in observed during loading is often considered to be the onset of plastic deformation $[36,37]$. Figure 6 shows typical pop-ins for these materials and uses a cumulative distribution of load at the first yield point (pop-in) to compare differences in defect density or defect formation. The ease with which 246 TFBA accommodates plastic deformation relative to PETN and erythritol indicates that either the initial defect density was very high in 246 TFBA, or that defects form easily in that material. However, given that all materials tested yield at loads below $1 \mathrm{mN}$, and loads expected in regular processing such as pressing are generally at least a thousand times higher than this [38], these differences are not expected to be impactful. In other words, relative to the high load conditions of a die press, the materials are effectively equally prone to plastic deformation.

Semi-quantitative measurements of fracture behavior are feasible using indentation testing; while there are challenges in extracting pure mode I fracture toughness, relative toughness assessments are possible by using indentation probes which cause a stress field to initiate and propagate a crack in the material surrounding the region being indented. As pelleting and compaction can cause both plastic deformation and fracture of individual crystals, assessing fracture behavior in as-grown materials provides a method to test for suitability of mock materials without the need for initial large volumes required to create pellets in conventional press/tableting. An unloading analysis technique to detect the occurrence of indentation fracture was previously developed by Morris et al. [23] and has been tested for its efficacy on brittle molecular crystals [24]. This technique is especially useful for materials which exhibit fracture at very low loads, where inspecting the material post-indentation is difficult to verify cracking. In the unloading analysis method, a material is indented with two probes of highly varied acuity to the same maximum load, and the unloading portions of the load-depth curves for those two indents are plotted together. Superimposability of these curves implies that indentation did not cause a fracture event, while nonsuperimposability implies indentation-induced fracture. As seen in Figure 7, PETN, erythritol, and 246 TFBA all experience indentation fracture with a cube corner probe geometry (the more acute of the two geometries, and thus the more likely to cause fracture) at loads as low as $500 \mu \mathrm{N}$. 


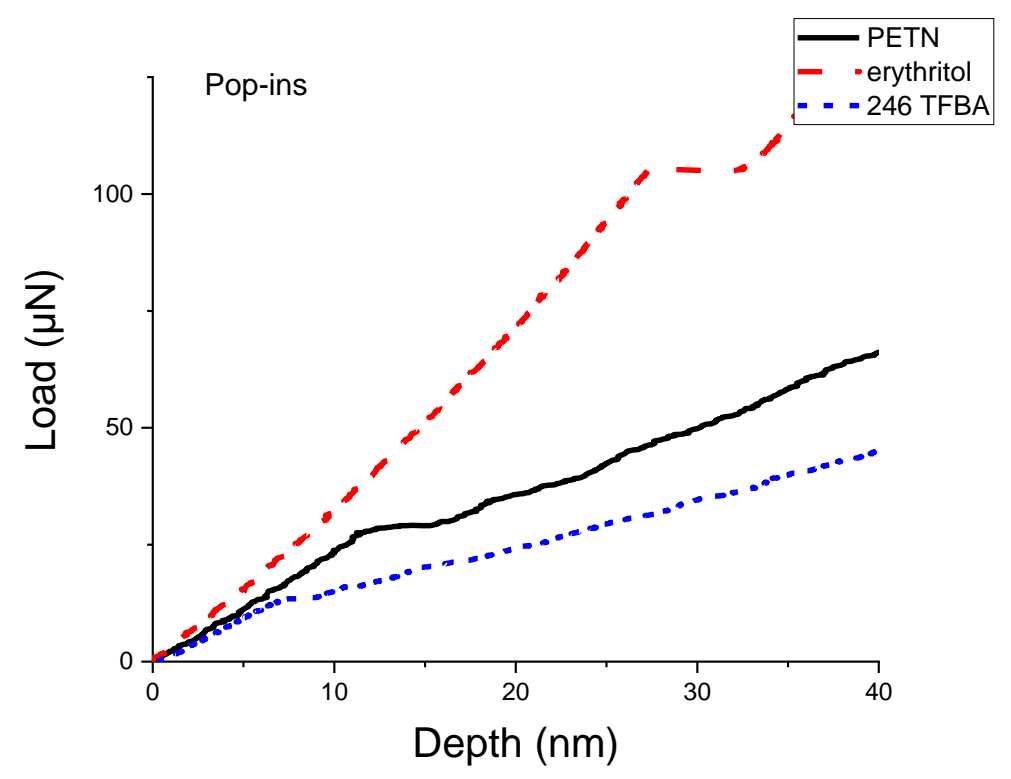

(a)

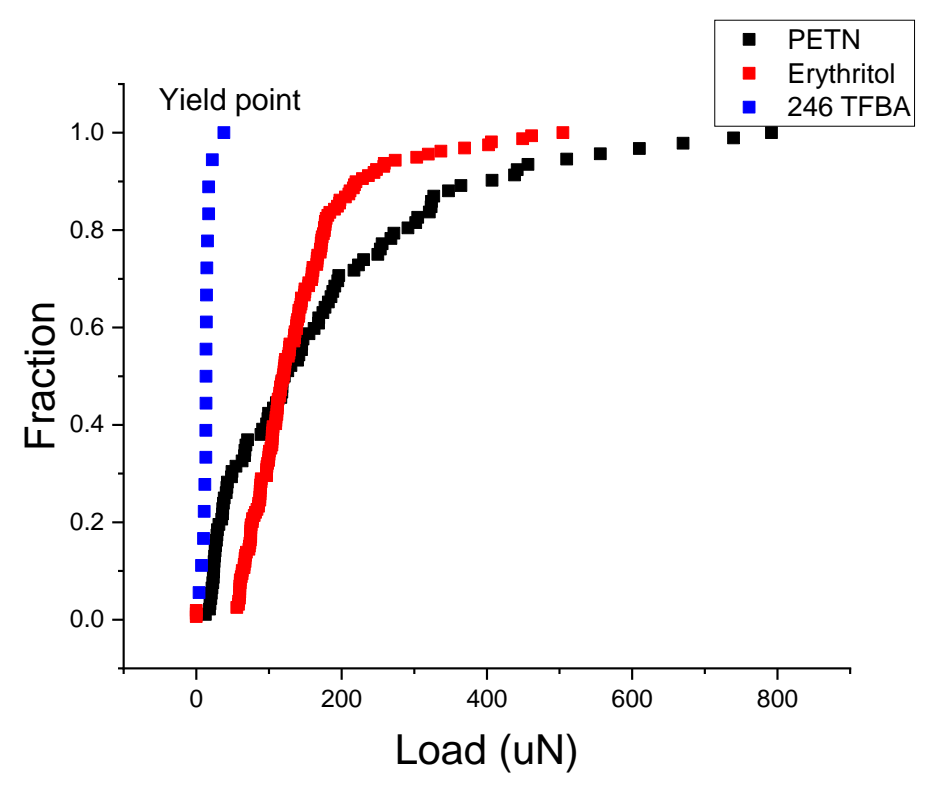

(b)

Figure 6. (a) Load-depth curve excerpts for erythritol, PETN, and 246 TFBA exhibiting pop-ins. (b) Cumulative fraction of yield behavior for erythritol, PETN, and 246 TFBA. Similar yield behavior is shown for PETN and erythritol, with mean load at yields of $175 \mu \mathrm{N}$ and $140 \mu \mathrm{N}$ respectively, whereas 246 TFBA shows a much lower resistance to plasticity with a mean load at yield of $15 \mu \mathrm{N}$. 


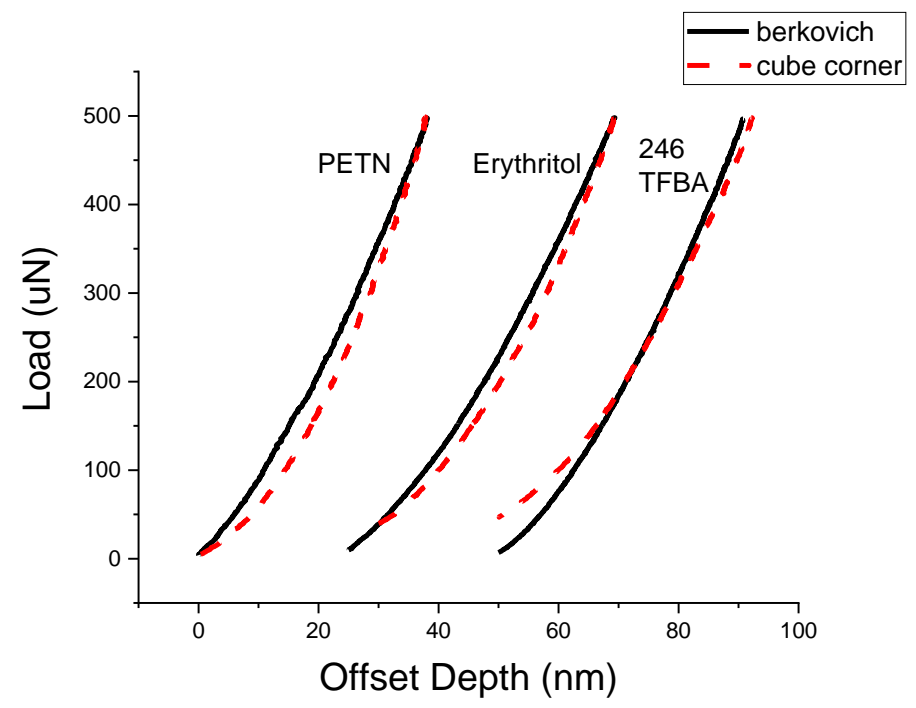

Figure 7. Unloading traces indicating indentation-induced fracture at $500 \mu \mathrm{N}$ for PETN, erythritol, and 246 TFBA.

Based on these results showing fracture in PETN, erythritol, and 246 TFBA with a cube corner probe at loads as low at $500 \mu \mathrm{N}$, the validity of Berkovich measurements on these materials at a maximum load of $1 \mathrm{mN}$ became a concern, as the method used for determining hardness and elastic modulus is only valid for indents in which a fracture event did not occur. In order to address these concerns, PETN was indented with a Berkovich probe at loads of $1 \mathrm{mN}$ intervals from $2 \mathrm{mN}$ to $12 \mathrm{mN}$ and scanning probe microscopy was used to check for evidence of cracking. Figure 8 shows typical images for indents at loads of $2 \mathrm{mN}, 4 \mathrm{mN}$, and $12 \mathrm{mN}$. The $12 \mathrm{mN}$ indent shows clear evidence of fracture while the $2 \mathrm{mN}$ and $4 \mathrm{mN}$ indents do not. Overall, fracture using a Berkovich tip was never observed in PETN at loads below $8 \mathrm{mN}$. This result provided confidence in measurements of modulus and hardness taken with a Berkovich probe of $1 \mathrm{mN}$.

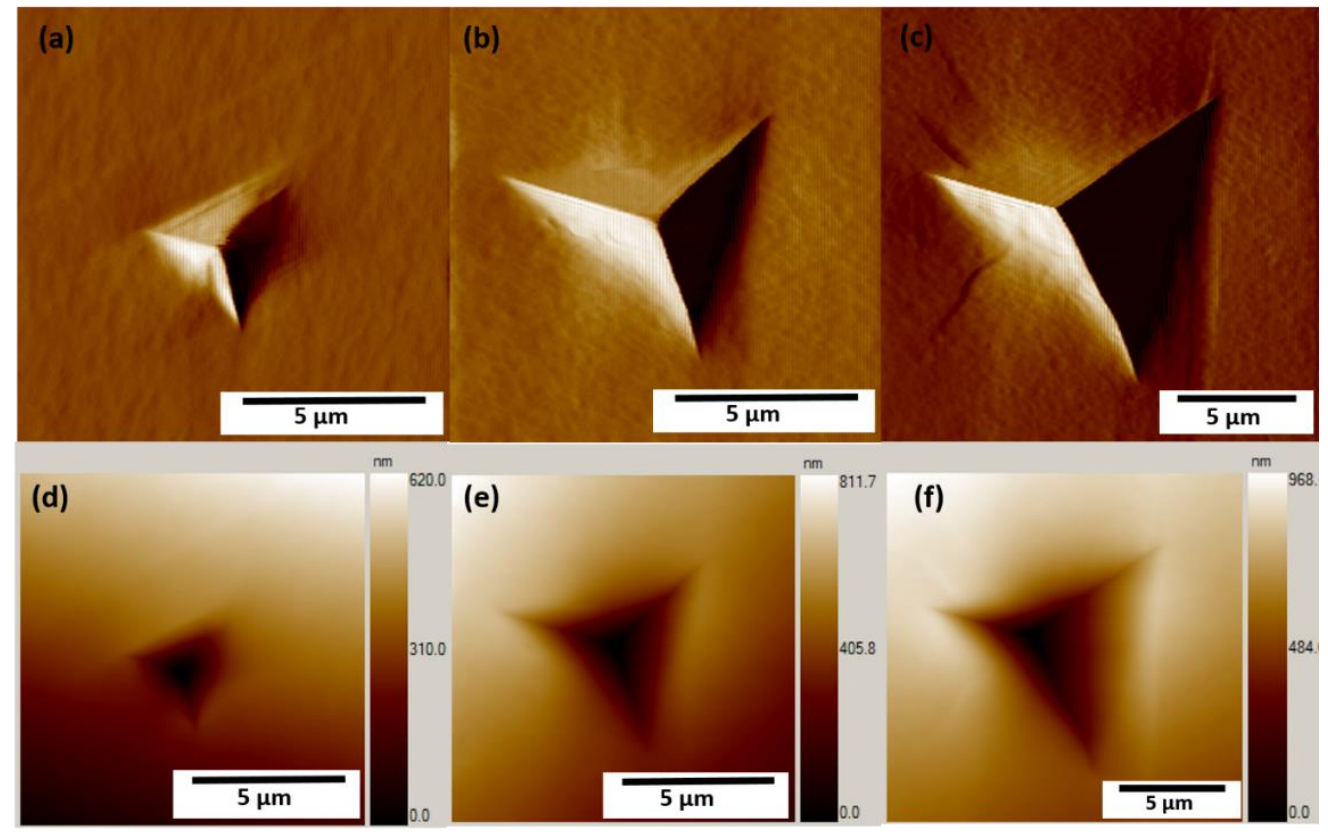

Figure 8. Scanning probe images (gradient) of Berkovich indents in PETN at loads of (a) $2 \mathrm{mN}$ with no evidence of fracture, (b) $4 \mathrm{mN}$ with no evidence of fracture, (c) $12 \mathrm{mN}$ with possible fracture visible, and $(\mathbf{d}-\mathbf{f})$ scanning probe images (topographical) of the same indents as $(\mathbf{a}-\mathbf{c})$. 


\subsection{Assessment of Experimental Uncertainty due to Crystal Properties and Anisotropy.}

Mechanical anisotropy between crystal faces of molecular crystals is well-documented [16,39,40] and possible preferred directions for fracture have been observed [24], indicating that there may be in-plane directional anisotropy that is sensitive to the relative normal orientation of pyramidal indenter probes with respect to the crystal face. For example, some researchers have found that cracks tend to nucleate from the corners of pyramidal indents due to stress localization [41]. To test for this on various crystals of both PETN and erythritol, a single face of the crystal was chosen to be indented, and an arbitrary direction of that face chosen as the origin, labeled $0^{\circ}$. Two probes were chosen to study any in-plane anisotropy. First, a typical pyramidal (Berkovich) tip was used, which has a $120^{\circ}$ angle between the edges. Second, an axisymmetric conical tip was used, with a circular cross section. The axisymmetric tip should show no in-plane orientation effects; if any were observed, the variation should only be due to random variability in the local properties. During the experiment, each crystal was rotated about a consistent normal direction relative to the arbitrary origin, and indented at $30^{\circ}$ intervals with both Berkovich and conical probes. The goal was to determine typical variability of elastic modulus and hardness as a function of indenter probe angle. This procedure is similar to that employed by Taw et al [13] for aspirin and Maughan et al [21] for griseofulvin, though those studies sampled only the one crystal morphology each, with only a single pyramidal probe. The results of the present orientation study, exemplified in Figure 9, were that the in-plane orientation of the indenter relative to the crystal surface did not seem to affect the measured properties. Some random indents had abnormally low or high modulus results due, most likely, to local material properties (e.g., defects), but there were no systematic trends, particularly trends that would repeat at orientations that would align with equivalent directions (i.e., (111) and (111). This finding provides confidence in all nanoindentation-derived mechanical properties measured to date in these highly anisotropic molecular crystal materials.

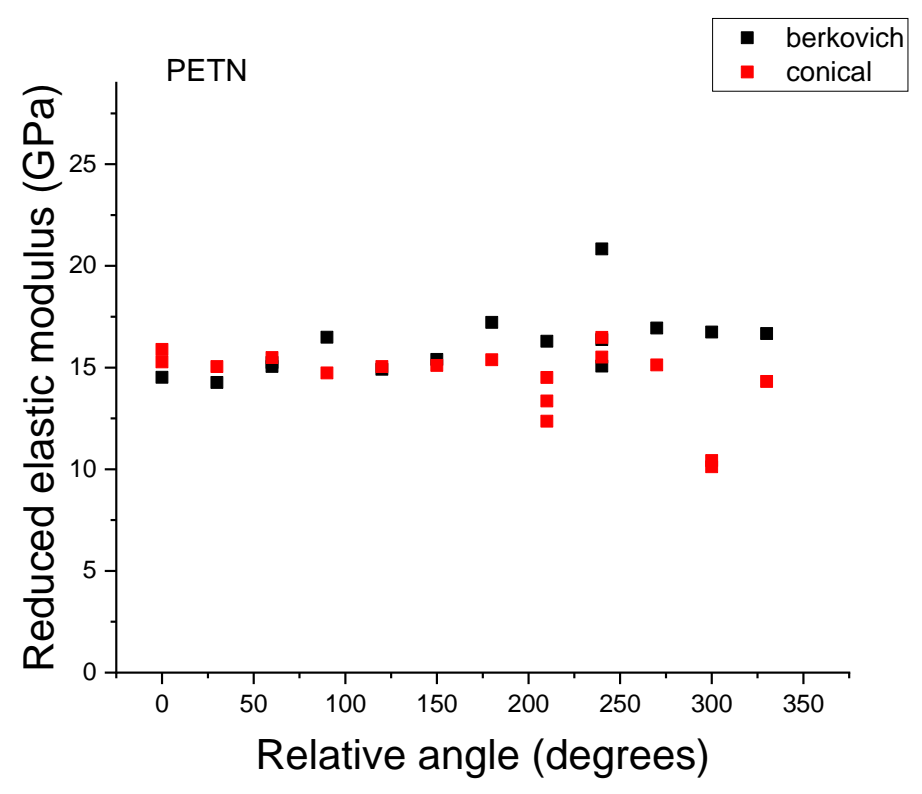

Figure 9. Cont. 


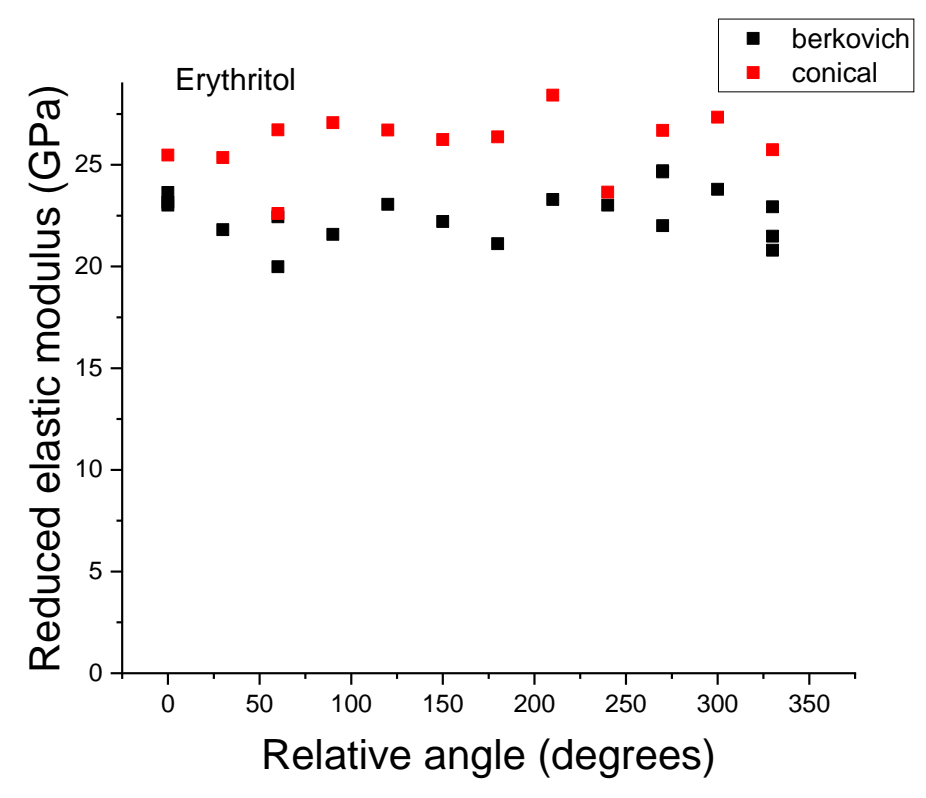

Figure 9. Elastic modulus of PETN and erythritol as a function of relative angle, showing no evidence of in-plane orientation dependence. Orientation is arbitrary between samples, so $0^{\circ}$ is not the same orientation between PETN and erythritol.

\section{Discussion}

The series of thermal and mechanical tests on PETN and several candidate mocks revealed that 246 TFBA was a promising material for generically mocking PETN in a variety of applications. It matched the melting point and density of PETN almost exactly, and was within acceptable range $(20 \%)$ of elastic modulus and hardness. It tended to plastically deform more easily than PETN, but for most applications both materials would be considered equally soft, and fracture behavior was effectively identical within the error of measurement. In other words, during quasi-static pressing or any other mechanically intensive operation, PETN and 246 TFBA should behave similarly. 246 TFBA is available commercially at reasonable, though not particularly cheap, prices. Overall, 246 TFBA is the most promising material to date for mocking a blend of PETN thermal and mechanical properties with acceptable density match.

Other mock materials investigated here could be used as surrogates for PETN in specific applications, depending on which properties were most important. Erythritol was found to be a reasonable thermomechanical mock, particularly in the similarity of the yield behavior, but the difference in density may be too large for some applications. Erythritol is widely available and relatively cheap, and so may be a cost-effective mock for PETN, especially for large-scale applications. N-BPFPO was an even better mechanical mock than erythritol but was much higher in density and currently requires on-demand synthesis to produce material. It also has a substantially higher melting point, potentially limiting applicability as a thermal mock. HFPT and PFBA are commercially available and match PETN hardness, but the thermal and elastic properties were quite far off, and so are not recommended for a PETN mock moving forward.

\section{Conclusions}

Of the five mock materials considered here as surrogates for PETN, several had reasonable matches to various PETN properties. 246 TFBA had the best blend of thermal and mechanical similarity to PETN, with nearly identical density and melting points and $<20 \%$ difference in mechanical properties. Erythritol may be a convenient alternative if matching PETN density is not important for the specific application, while N-BPFPO is a good choice if matching melt behavior is not important. Nanoindentation was found to be an especially useful tool to rapidly test these molecular crystals 
for basic mechanical properties, but was also highly informative for detailed plasticity and fracture measurements. Despite their high degree of anisotropy, further indentation tests revealed no in-plane orientation effects on measured properties, giving confidence in the results reported here and elsewhere in the literature on this class of material.

Author Contributions: A.C.B. and Z.R.W. conceptualized the experiments; A.C.B. performed all mechanical testing; J.D.Y. performed thermal analysis of the materials; J.D.Y. and D.F.B. designed some initial experiments and provided analysis of the data; Z.R.W. provided resources; final data analysis and curation was carried out by A.C.B.; all authors contributed to the writing and revisions of the paper. All authors have read and agreed to the published version of the manuscript.

Funding: Funding for this work was provided by the Delivery Environments Program at LANL under the direction of Antranik Siranosian. Los Alamos National Laboratory, an affirmative action equal opportunity employer, is operated by TRIAD National Security, LLC, for the National Nuclear Security Administration of the U.S. Department of Energy under Contract No. 89233218-NCNA000001.

Acknowledgments: The authors gratefully acknowledge Amanda Duque and Marvin Shorty (LANL) for helping to synthesize and crystallize the mock materials studied here, as well as Christian Sorenson and Steven Son (Purdue University) for synthesizing the PETN. The M-7 Analytical Team operated the DSC and helpfully provided data. This work was performed, in part, at the Center for Integrated Nanotechnologies, an Office of Science User Facility operated for the U.S. Department of Energy (DOE) Office of Science.

Conflicts of Interest: The authors declare no conflict of interest.

\section{References}

1. Matyáš, R.; Šelešovský, J.; Musil, T. Sensitivity to friction for primary explosives. J. Hazard. Mater. 2012, 213, 236-241. [CrossRef]

2. Gibbs, T.R.; Popolato, A. LASL Explosive Property Data; University of California: Berkeley, CA, USA, 1980.

3. Kubas, G.; Rees, W.; Caguiat, J.; Asch, D.; Fagan, D.; Cortes, P. Identification of peptide sequences that selectively bind to pentaerythritol trinitrate hemisuccinate-a surrogate of PETN, via phage display technology. Pept. Sci. 2017, 108, e22997. [CrossRef] [PubMed]

4. Konek, C.; Wilkinson, J.; Esenturk, O.; Heilweil, E.; Kemp, M. Terahertz spectroscopy of explosives and simulants: RDX, PETN, sugar, and L-tartaric acid. In Proceedings of the Terahertz Physics, Devices, and Systems III: Advanced Applications in Industry and Defense, Orlando, FL, USA, 30 April 2009; Volume 7311, p. $73110 \mathrm{~K}$.

5. Carter, J.C.; Angel, S.M.; Lawrence-Snyder, M.; Scaffidi, J.; Whipple, R.E.; Reynolds, J.G. Standoff detection of high explosive materials at 50 meters in ambient light conditions using a small Raman instrument. Appl. Spectrosc. 2005, 59, 769-775. [CrossRef] [PubMed]

6. Yeager, J.D.; Duque, A.L.H.; Shorty, M.; Bowden, P.R.; Stull, J.A. Development of inert density mock materials for HMX. J. Energy Mater. 2018, 36, 253-265. [CrossRef]

7. Williams, J.H.G.; McCool, A.A. Development of a Mechanically Matched Simulant for AFX-808: AFS-808; Air Force Research Laboratory: Montgomery County, OH, USA, 2014.

8. Ahmido, T.; Ting, A.; Misra, P. Femtosecond laser-induced breakdown spectroscopy of surface nitrate chemicals. Appl. Opt. 2013, 52, 3048-3057. [CrossRef]

9. Simpson, R.L.; Pruneda, C.O. Non-Detonable and Non-Explosive Explosive Simulators. U.S. Patent 5,648,636, 15 July 1997.

10. Dobratz, B.M. Properties of Chemical Explosives and Explosive Simulants; University of California: Berkeley, CA, USA; Lawrence Livermore Lab.: Livermore, CA, USA, 1972.

11. Liu, C.; Rae, P.J.; Cady, C.M.; Lovato, M.L. Damage \& fracture of high-explosive mock subject to cyclic loading. In Mechanics of Time-Dependent Materials and Processes in Conventional and Multifunctional Materials; Springer: Berlin, Germany, 2011; Volume 3, pp. 151-157.

12. Sheffield, S.A.; Gustavsen, R.L.; Alcon, R.R. Porous HMX initiation studies-sugar as an inert simulant. AIP Conf. Proc. 1998, 429, 575-578.

13. Taw, M.R.; Yeager, J.D.; Hooks, D.E.; Carvajal, T.M.; Bahr, D.F. The mechanical properties of as-grown noncubic organic molecular crystals assessed by nanoindentation. J. Mater. Res. 2017, 32, 2728-2737. [CrossRef] 
14. Taw, M.R.; Bahr, D.F. The Mechanical Properties of Minimally Processed RDX. Propellants Explos. Pyrotech. 2017, 42, 659-664. [CrossRef]

15. Burch, A.; Yeager, J.; Bahr, D. Nanoindentation of HMX and Idoxuridine to Determine Mechanical Similarity. Crystals 2017, 7, 335. [CrossRef]

16. Ramos, K.J.; Hooks, D.E.; Bahr, D.F. Direct observation of plasticity and quantitative hardness measurements in single crystal cyclotrimethylene trinitramine by nanoindentation. Philos. Mag. 2009, 89, 2381-2402. [CrossRef]

17. Mannepalli, S.; Mangalampalli, K. Indentation Plasticity and Fracture Studies of Organic Crystals. Crystals 2017, 7, 324. [CrossRef]

18. Zhai, M.; McKenna, G.B. Mechanical properties of pentaerythritol tetranitrate(PETN) single crystals from nano-indentation: Depth dependent response at the nano meter scale. Cryst. Res. Technol. 2016, 51, 414-427. [CrossRef]

19. Groom, C.R.; Bruno, I.J.; Lightfoot, M.P.; Ward, S.C. The Cambridge structural database. Acta Crystallogr. Sect. B 2016, 72, 171-179. [CrossRef]

20. Yamaguchi, K.; Matsumura, G.; Haga, N.; Shudo, K. Structure of N, N-bis (2,3,4,5,6-pentafluorophenyl) oxamide. Acta Crystallogr. Sect. C 1992, 48, 558-559. [CrossRef]

21. Maughan, M.R.; Carvajal, M.T.; Bahr, D.F. Nanomechanical testing technique for millimeter-sized and smaller molecular crystals. Int. J. Pharm. 2015, 486, 324-330. [CrossRef]

22. Oliver, W.C.; Pharr, G.M. An improved technique for determining harness and elastic modulus using load and displacement sensing indentation experiments. J. Mater. Res. 1992, 7, 1564-1583. [CrossRef]

23. Morris, D.J.; Myers, S.B.; Cook, R.F. Sharp probes of varying acuity: Instrumented indentation and fracture behavior. J. Mater. Res. 2004, 19, 165-175. [CrossRef]

24. Burch, A.C.; Yeager, J.D.; Bahr, D.F. Indentation fracture behavior of energetic and inert molecular crystals. J. Mater. Res. 2019, 34, 3954-3963. [CrossRef]

25. Yeager, J.D.; Woznick, C.S.; Thompson, D.G.; Duque, A.L.; Liu, C.; Burch, A.C.; Bowden, P.R.; Shorty, M.; Bahr, D.F. Development of a new density and mechanical mock for HMX. In Proceedings of the Sixteenth International Detonation Symposium, Cambridge, MD, USA, 16 July 2018; p. 1631.

26. Shimada, A. Crystal and molecular structure of mesoerythritol. Acta Crystallogr. 1958, 11, 748-749. [CrossRef]

27. Manner, V.W.; Tappan, B.C.; Scott, B.L.; Preston, D.N.; Brown, G.W. Crystal structure, packing analysis, and structural-sensitivity correlations of erythritol tetranitrate. Cryst. Growth Des. 2014, 14, 6154-6160. [CrossRef]

28. Cady, H.H.; Larson, A.C. Pentaerythritol tetranitrate II: Its crystal structure and transformation to PETN I; an algorithm for refinement of crystal structures with poor data. Acta Crystallogr. Sect. B 1975, 31, 1864-1869. [CrossRef]

29. Betz, R.; Gerber, T. 2,4,6-Trifluorobenzoic acid. Acta Crystallogr. Sect. E 2011, 67, o539. [CrossRef]

30. Shimada, A. Crystal structure and lattice energy of i-erythritol. i. crystal structure of i-erythritol. Bull. Chem. Soc. Jpn. 1959, 32, 325-329. [CrossRef]

31. Bouwman, E.; Caulton, K.G.; Christou, G.; Folting, K.; Gasser, C.; Hendrickson, D.N.; Huffman, J.C.; Lobkovsky, E.B.; Martin, J.D. Doubly-hydrated hexafluoroacetylacetone as a tetradentate ligand: Synthesis, magnetochemistry, and thermal transformations of a dimanganese (III) complex. Inorg. Chem. 1993, 32, 3463-3470. [CrossRef]

32. Gdaniec, M. 2,3,4,5,6-Pentafluorobenzamide. Acta Crystallogr. Sect. E Struct. 2003, 59, o1642-o1644. [CrossRef]

33. Maiti, A.; Gee, R.H. PETN Coarsening-Predictions from Accelerated Aging Data. Propellants Explos. Pyrotech. 2011, 36, 125-130. [CrossRef]

34. Maiti, A.; Han, Y.; Zaka, F.; Gee, R.H. In-situ Monitoring of Flow-Permeable Surface Area of High Explosive Powder using Small Sample Masses. Propellants Explos. Pyrotech. 2015, 40, 419-425. [CrossRef]

35. Ramos, K.J.; Bahr, D.F.; Hooks, D.E. Defect and surface asperity dependent yield during contact loading of an organic molecular single crystal. Philos. Mag. 2011, 91, 1276-1285. [CrossRef]

36. Bahr, D.F.; Kramer, D.E.; Gerberich, W.W. Non-linear deformation mechanisms during nanoindentation. Acta Mater. 1998, 46, 3605-3617. [CrossRef]

37. Mann, A.B.; Pethica, J.B. The role of atomic size asperities in the mechanical deformation of nanocontacts. Appl. Phys. Lett. 1996, 69, 907-909. [CrossRef] 
38. Monroe, D.C. (U) Historical PETN Pressing Data: 1965-1978; Los Alamos National Lab. (LANL): Los Alamos, NM, USA, 2015.

39. Ramos, K.J.; Bahr, D.F. Mechanical behavior assessment of sucrose using nanoindentation. J. Mater. Res. 2007, 22, 2037-2045. [CrossRef]

40. Zhou, X.; Lu, Z.; Zhang, Q.; Chen, D.; Li, H.; Nie, F.; Zhang, C. Mechanical anisotropy of the energetic crystal of 1,1-diamino-2,2-dinitroethylene (FOX-7): A study by nanoindentation experiments and density functional theory calculations. J. Phys. Chem. C 2016, 120, 13434-13442. [CrossRef]

41. Cook, R.F.; Pharr, G.M. Direct Observation and Analysis of Indentation Cracking in Glasses and Ceramics. J. Am. Ceram. Soc. 1990, 73, 787-817. [CrossRef]

(C) 2020 by the authors. Licensee MDPI, Basel, Switzerland. This article is an open access article distributed under the terms and conditions of the Creative Commons Attribution (CC BY) license (http://creativecommons.org/licenses/by/4.0/). 